\title{
Marine Safety and Data Analytics: Vessel Crash Stop Maneuvering Performance Prediction
}

\author{
Luca Oneto $^{1}$, Andrea Coraddu ${ }^{2}$, Paolo Sanetti ${ }^{1}$, Olena Karpenko ${ }^{3}$, \\ Francesca Cipollini ${ }^{1}$, Toine Cleophas ${ }^{3}$, and Davide Anguita ${ }^{1}$ \\ 1 DIBRIS - University of Genoa, Italy \\ \{luca.oneto, paolo.sanetti, francesca.cipollini, davide.anguita\}@unige.it \\ 2 School of Marine Science \& Technology \\ Newcastle University, United Kingdom \\ andrea. coraddu@newcastle.ac.uk \\ 3 DAMEN Shipyards Gorinchem, The Netherlands \\ \{olena.karpenko, toine.cleophas\}@damen.com
}

\begin{abstract}
Crash stop maneuvering performance is one of the key indicators of the vessel safety properties for a shipbuilding company. Many different factors affect these performances, from the vessel design to the environmental conditions, hence it is not trivial to assess them accurately during the preliminary design stages. Several first principal equation methods are available to estimate the crash stop maneuvering performance, but unfortunately, these methods usually are either too costly or not accurate enough. To overcome these limitations, the authors propose a new data-driven method, based on the popular Random Forests learning algorithm, for predicting the crash stopping maneuvering performance. Results on real-world data provided by the DAMEN Shipyards show the effectiveness of the proposal.
\end{abstract}

Keywords: Marine Safety, Vessel Maneuvering, Crash Stop, Data-Driven Methods, Random Forests, Performance Assessment, Performance Estimation

\section{Introduction}

Shipping is one of the most safety critical industry [15]. For this reason, as reported in $[17,16]$, the vessel's design has to ensure that the craft should be controllable and be capable of maneuvering securely up to the critical design conditions. In this paper, the authors focus their attention on a particular safety related maneuver which is the crash stop. The goal is to predict, at design stage, the crash stop main characteristics, for the preliminary assessment of safety requirement imposed by the classification society [14]. The crash stop maneuvering is usually performed to avoid any collision or crashing of a ship into any other ship or structure. During this maneuver, the main engine is subjected to severe stress and loading since it involves slowing, stopping and reversing the direction as fast as possible.

The assessment of the ship crash stop maneuver plays a crucial role in the marine engineering field. Several first principal equation methods are available to estimate the crash stop performance $[18,20,21,12,25]$, but the latter are either 
too complex $[18,25]$ or not accurate enough [20] due to the complexity of the system. To increase their accuracy, several parameters need to be provided by the different manufacturer of the vessel components, and finally, models need to be fine tuned based on the outcomes of several sea trials which makes the process costly, time-consuming, and not applicable at design stage [18]. Moreover, suppliers are usually not willing to share technical details which may harm their industrial competitive advantages.

In this paper, the authors propose a new fully data-driven method based on Random Forests (RF), a state-of-the-art powerful learning algorithm, first developed in [4] and then recently improved in [3], for predicting the crash stopping performance. Data-driven methods, instead of relying on the physical knowledge about the system, build upon historical data collected about a phenomenon to build a model which can easily take into account many different sources of information which cannot be easily modeled with first principle equations. RF are usually preferred to other classification techniques, because of their high numerical robustness, their innate capacity of dealing with numerical and categorical features, and their effectiveness in many real-world problems [10,24]. By carefully tuning the RF hyperparameters [22] and by assessing the performance of the final learned model with state-of-the-art resampling techniques [2], authors will show the effectiveness of the proposal.

In summary, the paper contribution is twofold. From a marine engineering perspective, the paper deals with the problem of the prediction of the crash stop main characteristics without taking into account the physical laws that are governing the phenomenon. In fact, authors proposal does not require any apriory knowledge about the problems and allows to exploit information sources which cannot be modeled with conventional approaches. From a data analytics perspective, this paper proposes an alternative RF formulation and shows that a careful tuning procedure of the RF hyperparameters can remarkably improve its performance. Results on real-world data coming from the DAMEN Shipyards demonstrate the effectiveness of the proposal. In particular, DAMEN, in its many years of vessels production, conducted several sea trials to measure vessels general seaworthiness and performance. For this application, the authors used a particular cluster of DAMEN vessels, the High-Speed Craft family [8], as a test case.

\section{Vessel Crash Stop Maneuvering}

As reported in [1] the stopping ability of a vessel is measured by three main parameters: the Track Reach (TR), the Head Reach (HR) and the Time for Full Maneuver (or time to dead in water) TFM. Also the Lateral Deviations (LD), Lateral Deviation Direction (LDD), and Heading Deviation Direction (HDD) are parameters of interest, but they are more sensitive to initial conditions and wind disturbances. The crash stop maneuver consists in a stop engine full astern performed after a steady approach at the test speed until the vessel starts going backwards. TR is defined as a distance along the vessel track that the vessel covers from the moment that the full astern command is given until ahead speed changes sign. The HR, instead, is the distance along the direction of the course at the moment when the full astern command is given. The distance is measured from the moment when the full astern command is given until the vessel is 
stopped dead in the water. The LD is defined as the distance perpendicular to the direction of the course at the moment when the full astern command is given. Also this distance is measured from the moment when the full astern command is given until the vessel is stopped dead in the water. Figure 1 shows the meaning of each parameter.

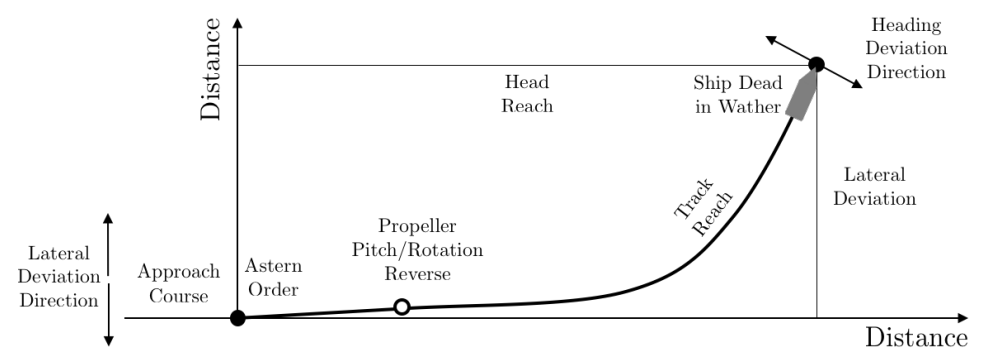

Fig. 1: Crash Stop maneuver performance indexes.

The main parameters of the crash stop maneuver are evaluated by means of the full-scale trials. At design stage, in order to assess the maneuvering characteristics both in trail and full load conditions, reliable methods should be applied. These methods should ensure satisfactory accuracies for the prediction of new vessels and satisfactory extrapolation of trial results to the full load condition. As reported in [12], the factors which affect the stopping ability of vessels are the vessel displacement, the initial speed, the block coefficient, the vessel hull fouling degree, the main engine full astern power, the time taken to effect changes in engine telegraph settings, the propeller category, and the environmental conditions (e.g. wind, stream, and the depth of water). During this maneuvers, the interactions between hull and propeller(s) are quite complex to be modeled. For this reason, empirical calculations of its characteristics are used when adequate motion equation coefficients are not available for simulation $[18,20]$. In this paper the authors focused their attention only on the crash stop from maximum operational speed and any transient mode speed according to the available data provided to DAMEN Shipyards. To prove the effectiveness of the new datadriven approach, authors focused on a particular cluster of DAMEN vessels, the High-Speed Craft (HSC) family [8], but the method is general and the data can be easily retrieved by any shipbuilder. The total amount of the vessels is 230 divided into four product clusters: Fast Crew Supplier, Search And Rescue, Stan Pilot and Stan Tender. For each product cluster, different products (vessel type) are available, each one characterized by several yard numbers. Every single yard number can perform several crash stop maneuvers. For each of the vessels, the information reported in Table 1 is available. During the trials, the maneuvers are digitally recorded using advanced portable measurement equipment. Therefore, the goal is to predict, based on the information of Table 1, available at design stages, the crash stop maneuver performance indexes (TR, HR, TFM, LD, LDD, and HDD).

\section{Proposed Data Driven Approach}

In this section, the authors will present the proposed data-driven vessel crash stop maneuvering performance prediction system. 
Table 1: Available vessels information.

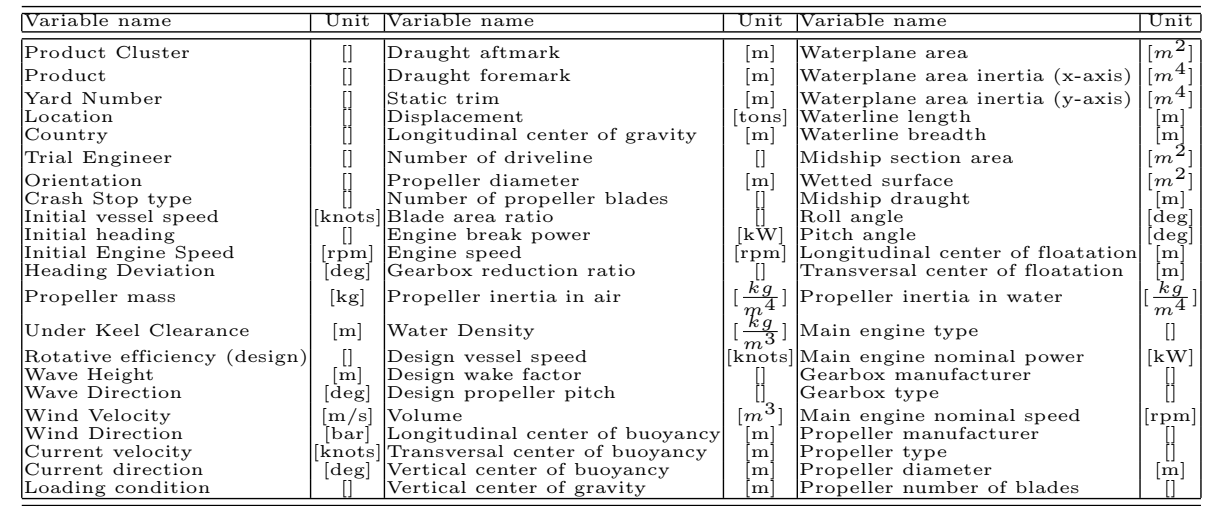

Let authors consider the supervised learning framework where an input space $\mathcal{X}=\mathcal{X}_{1} \times \cdots \times \mathcal{X}_{d}$, composed of $d$ features, and an output space $\mathcal{Y}$ are available [23]. $\mathcal{X}_{i}$ can be a categorical feature space $\mathcal{X}_{i}=\left\{c_{i}^{1}, \cdots, c_{i}^{n_{c_{i}}}\right\}$ or a real valued feature space $\mathcal{X}_{i} \subseteq \mathbb{R}$ (see Table 1 ). Analogously, also $\mathcal{Y}$ can be a binary valued output space $\mathcal{Y}=\{ \pm 1\}$ (LDD and HSS) or real valued output space $\mathcal{Y} \subseteq \mathbb{R}(\mathrm{TR}, \mathrm{HR}, \mathrm{TFM}$, and LD). Based on the type of output space the associated learning problem is called binary classification or regression respectively [23]. In the supervised learning framework, the goal is to estimate the unknown rule $\mu: \mathcal{X} \rightarrow \mathcal{Y}$ which associates an element $Y \in \mathcal{Y}$ to an element $X \in \mathcal{X}$. Note that, in general, $\mu$ can be non-deterministic [23] and some components of $X$ may be missing (errors in the measurements, careless operators, etc.) [9]. In this case, if the missing value is located in a categorical feature, an additional category for missing values is introduced on those features. If, instead, the missing value is associated to a numerical feature, as suggested in [9], that missing value is replaced with the mean value of that feature and an additional logical feature is introduced to indicate if the value of that feature for a particular sample is missing or not. An ML technique estimates $\mu$ through a learning algorithm $\mathscr{A}_{\mathcal{H}}: \mathcal{D}_{n} \times \mathcal{F} \rightarrow f$, characterized by its set of hyperparameters $\mathcal{H}$, which maps a series of examples of the input/output relation, contained in a datasets of $n$ samples $\mathcal{D}_{n}:\left\{\left(X_{1}, Y_{1}\right), \cdots,\left(X_{n}, Y_{n}\right)\right\}$ sampled i.i.d from $\mu$, into a function $f: \mathcal{X} \rightarrow \mathcal{Y}$. The error that $f$ commits, in approximating $\mu$, is measured with reference to a loss function $\ell: \mathcal{X} \times \mathcal{Y} \times \mathcal{F} \rightarrow[0, \infty)$ through the empirical error. Note that, for binary classification problems, authors will make use of the hard loss function which counts the number of errors $\ell_{\mathrm{H}}(f(X), Y)=[f(X) \neq Y] \in\{0,1\}[23]$ and for regression the truncated relative absolute error $\ell_{\mathrm{TRAE}}(f(X), Y)=\min [1,|f(X)-Y| /|Y|] \in[0,1]$ will be exploited [7]. The purpose of any learning procedure is to select the best set of hyperparameters $\mathcal{H}$ such that the expected error $L(f)=\mathbb{E}_{\mu} \ell(f(X), Y)$, which unfortunately is unknown since $\mu$ is unknown, is minimum. Since $L(f)$ is unknown, the empirical error $\widehat{L}^{\mathcal{D}_{n}}(f)=1 / n \sum_{(X, Y) \in \mathcal{D}_{n}} \ell(f(X), Y)$ must be exploited in order to estimate it.

In this paper $\mathscr{A}$ is a RF because of their high numerical robustness, native capacity of dealing with numerical and categorical features, and effectiveness in 
many real-world classification problems $[4,3]$. The original RF learning phase of each of the $n_{t}$ trees $\left\{T_{1}, \cdots, T_{n_{t}}\right\}$ composing the RF is quite simple [4]. From $\mathcal{D}_{n},\lfloor b n\rfloor$ samples are sampled with replacement and $\mathcal{D}_{\lfloor b n\rfloor}^{\prime}$ is built. A tree is constructed with $\mathcal{D}_{\lfloor b n\rfloor}^{\prime}$ but the best split is chosen among a subset of $n_{v}$ features over the possible $d$ features randomly chosen at each node. The tree is grown until the node contains a maximum of $n_{l}$ samples. During the classification phase of a previously unseen $X$, each tree classifies $X$ in a class $Y_{i \in\left\{1, \cdots, n_{t}\right\}}$, and then the final classification is the $\left\{p_{1}, \cdots, p_{n_{t}}\right\}$-weighted combination of all the answers of each tree of the RF. The empirical error of the tree $T$ built based on $\mathcal{D}_{\lfloor b n\rfloor}^{\prime}$ over the out of bag data $\mathcal{D}_{n} \backslash \mathcal{D}_{\lfloor b n\rfloor}^{\prime}$ is defined as $\widehat{L}^{\mathrm{oob}}(T) . p_{\left\{i \in 1, \cdots, n_{t}\right\}}$ are of paramount importance for the accuracy of an ensemble classifier $[19,11]$ and for this reason authors will exploit a state-of-the-art alternative proposed in [5] and recently further developed in $[19,22]$ where $p_{i}=e^{-\gamma \widehat{L}^{\circ o b}(T)}$ with $\gamma \in[\gamma, \infty)$. If $\gamma=0$, $b=1, n_{v}=\sqrt{n}$, and $n_{l}=1$ we get the original RF formulation [4]. RF have been recently improved in [3] which proposes to avoid the initial bootstrapping and the subset feature selection at each node construction of the trees by replacing it with a random rotation of the numerical feature space before learning each tree of the forest. Note that, since rotations can be sensitive to scale in general and to outliers in particular, the RF developed in [3] need to scale the numerical feature space. As suggested by the results in [3], the simple scaling of each feature in the range $[0,1]$ should be adopted. In this paper, authors propose to use the RF learning algorithm reported in Algorithm 1 which merges the original RF formulation [4] with the most recent one of [3]. In particular, authors propose to use the learning strategy proposed in [4] by also including the random rotation proposed in [3] in order to get the benefits of both approaches. Note that in Algorithm 1 the rotation does not change at each tree but every $n_{r}$ trees in order to reduce the computational requirements of the $\mathrm{RF}$ with respect to [3].

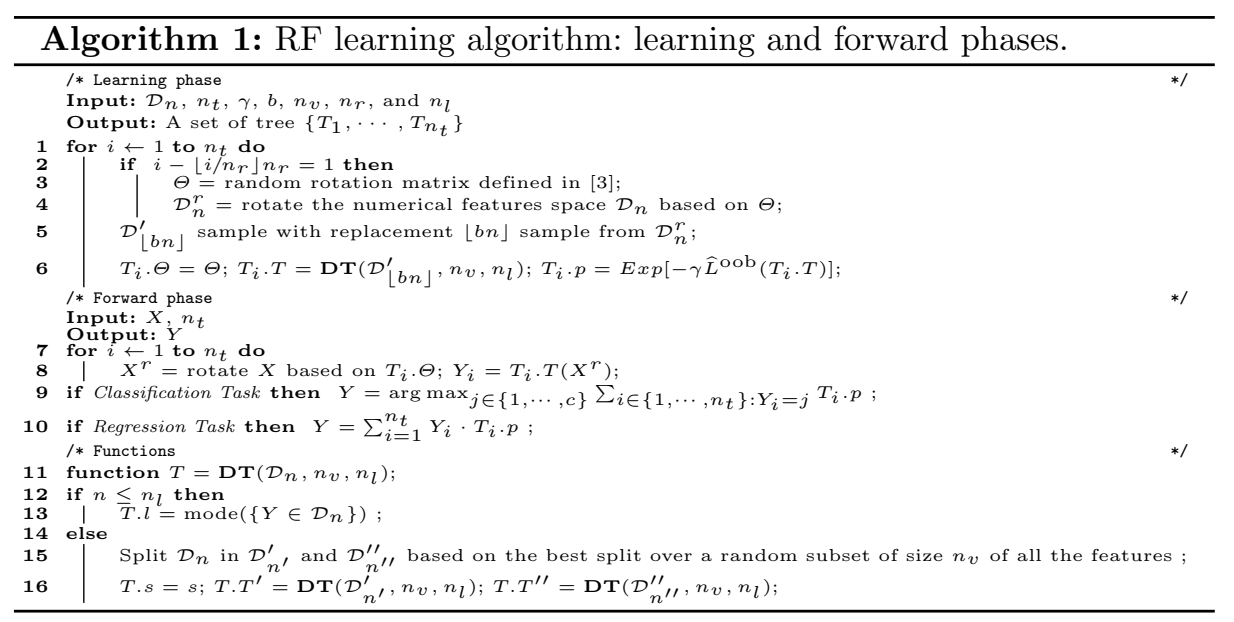

In order to tune, in a data dependent manner, the different hyperparameters of the RF of Algorithm 1 and to estimate the performance of the final model, the nonparametric Bootstrap (BOO) is exploited [2]. BOO relies on a simple idea: the original dataset $\mathcal{D}_{n}$ is resampled once or many $\left(n_{o}\right)$ times with replace- 
ment, to build three independent datasets called training, validation, and test sets, respectively $\mathcal{L}_{l}^{o}, \mathcal{V}_{v}^{o}$, and $\mathcal{T}_{t}^{o}$, with $o \in\left\{1, \cdots, n_{o}\right\}$. Note that $\mathcal{L}_{l}^{o} \cap \mathcal{V}_{v}^{o}=\oslash$, $\mathcal{L}_{l}^{o} \cap \mathcal{T}_{t}^{o}=\oslash$, and $\mathcal{V}_{v}^{o} \cap \mathcal{T}_{t}^{o}=\oslash$. Then, in order to select the best set of hyperparameters $\mathcal{H}$ in the set of possible ones $\mathfrak{H}=\left\{\mathcal{H}_{1}, \mathcal{H}_{2}, \cdots\right\}$ for the algorithm $\mathscr{A}_{\mathcal{H}}$ or, in other words, to perform the performance tuning phase, the following procedure needs to be applied:

$$
\mathcal{H}^{*}: \arg \min _{\mathcal{H} \in \mathfrak{H}} \frac{1}{n_{o}} \sum_{o=1}^{n_{o}} \widehat{L}^{\mathcal{V}_{v}^{o}}\left(\mathscr{A}_{\mathcal{H}}\left(\mathcal{L}_{l}^{o}\right)\right) .
$$

Since the data in $\mathcal{L}_{l}^{o}$ are i.i.d. with respect to the ones in $\mathcal{V}_{v}^{o}$, the idea is that $\mathcal{H}^{*}$ should be the set of hyperparameters which allows to achieve a small error on a data set that is independent from the training set. The uncertainty quantification, instead, is performed as follows $[2,13]$ :

$$
L\left(\mathscr{A}_{\mathcal{H}^{*}}\left(\mathcal{D}_{n}\right)\right) \leq \widehat{L}\left(\mathscr{A}_{\mathcal{H}^{*}}\left(\mathcal{D}_{n}\right)\right)+\sqrt{\frac{\log \left(\frac{1}{\delta}\right)}{2 t}}, \widehat{L}\left(\mathscr{A}_{\mathcal{H}^{*}}\left(\mathcal{D}_{n}\right)\right)=\frac{1}{n_{o}} \sum_{o=1}^{n_{o}} \widehat{L}^{\mathcal{T}_{t}^{o}}\left(\mathscr{A}_{\mathcal{H}^{*}}\left(\mathcal{L}_{l}^{o} \cup \mathcal{V}_{v}^{o}\right)\right)
$$

where the bound holds with probability $(1-\delta)$. Note that after the best set of hyperparameters is found, one can select the best model by training the algorithm with the whole data set $\mathscr{A}_{\mathcal{H}^{*}}\left(\mathcal{D}_{n}\right)$ [2] and since the data in $\mathcal{L}_{l}^{o} \cup \mathcal{V}_{v}^{o}$ are i.i.d. with respect to $\mathcal{T}_{t}^{o}$ it follows that $\widehat{L}^{\mathcal{T}_{t}^{o}}\left(\mathscr{A}_{\mathcal{H}^{*}}\left(\mathcal{L}_{l}^{o} \cup \mathcal{V}_{v}^{o}\right)\right)$ is an unbiased estimator of $L\left(\mathscr{A}_{\left(\mathcal{D}_{n}, \mathcal{H}^{*}\right)}\right)$. Then, any concentration result can be used, like the Hoeffding inequality [13], for bounding the bias between the expected value and its empirical estimator. Note that, in the BOO, $l=n$ and $\mathcal{L}_{l}^{o}$ must be sampled with replacement from $\mathcal{D}_{n}$, while $\mathcal{V}_{v}^{o}$ and $\mathcal{T}_{t}^{o}$ are sampled without replacement from $\mathcal{D}_{n} \backslash \mathcal{L}_{l}^{o}$.

Finally, note that in this paper authors set $n_{t}$ as large as possible. Since the performance of the $\mathrm{RF}$ always increases by increasing $n_{t}[4,3]$ we stop increasing $n_{t}$ when the performance of the RF stops to increase.

\section{Results}

In this section, authors report the results of applying the techniques proposed in Section 3 to the problem described in Section 2, based on the data provided by DAMEN Shipyards and outlined in the same section. In particular three approaches have been compared:

- ORF: the original RF proposed in [4];

- RFR: the RF proposed in [3] which improve over the ORF;

- PRF: the RF algorithms proposed in this paper (see Algorithm 1) where their hyperparameters have been tuned with the BOO procedure described in Section 3.

For what concerns PRF authors set $\mathcal{H}=\left\{\gamma, b, n_{v}, n_{r}, n_{l}\right\}$ and $\mathfrak{H}=\left\{10^{-4.0}, 10^{-3.5}\right.$, $\left.\cdots, 10^{3.0}\right\} \times\{0.7,0.8, \cdots, 1.2\} \times d^{\{0.0,0.1, \cdots, 1\}} \times\{1,10,100\} \times n \cdot\{0.0,0.01,0.05,0.1\}$, $n_{o}=100$ in the BOO procedure, and $n_{t}=10^{3}$ since larger values did not produce any improvement in the accuracies of ORF, RFR, and PRF in any of the experiments.

Moreover, three different scenarios have been investigated:

- S1: authors kept different yard number in each of the sets $\mathcal{L}_{l}^{o}, \mathcal{V}_{v}^{o}$, and $\mathcal{T}_{t}^{o}$. In this way in the training set both examples of different products and different product clusters are present; 
- S2: authors kept different products in each of the sets $\mathcal{L}_{l}^{o}, \mathcal{V}_{v}^{o}$, and $\mathcal{T}_{t}^{o}$. In this way the case when a new product needs to be designed is simulated;

- S3: authors kept different product clusters in each of the sets $\mathcal{L}_{l}^{o}, \mathcal{V}_{v}^{o}$, and $\mathcal{T}_{t}^{o}$. In this way, the case when a new series of products needs to be designed is simulated.

Note that $\mathrm{S} 1$ is a simpler task with respect to $\mathrm{S} 2$, which is again a simpler task with respect to S3. In fact, authors try to simulate the increasingly difficult task to extrapolate the performance indexes of a vessel, which is more and more different with respect to the vessels contained in the training set.

In Table 2 is reported $\widehat{L}\left(\mathscr{A}_{\mathcal{H}^{*}}\left(\mathcal{D}_{n}\right)\right)$ and $L\left(\mathscr{A}_{\mathcal{H}^{*}}\left(\mathcal{D}_{n}\right)\right)$ in percentage respectively for ORF, RFR, and PRF in S1, S2, and S3 where $\delta=0.05$. From the results it is possible to observe that:

- PRF mostly outperform ORF and RFR as expected;

- $\widehat{L}\left(\mathscr{A}_{\mathcal{H}^{*}}\left(\mathcal{D}_{n}\right)\right)$ and $L\left(\mathscr{A}_{\mathcal{H}^{*}}\left(\mathcal{D}_{n}\right)\right)$ are close with each other and this means that it is possible to guarantee a quality of the estimation which is close enough to the expected quality of the produced data-driven model;

- as expected, the performances in S1 are better than the ones in S2 and S3. Nevertheless, even in S2 an S3 the performances of PRF are quite satisfying since the errors are around $5 \%$. Note, instead, that for S3, ORF and RFR cannot be used in a real-world application because of their low accuracies.

Table 2: $\widehat{L}\left(\mathscr{A}_{\mathcal{H}^{*}}\left(\mathcal{D}_{n}\right)\right)$ and $L\left(\mathscr{A}_{\mathcal{H}^{*}}\left(\mathcal{D}_{n}\right)\right)$ of ORF, RFR, PRF in S1, S2, S3 (in \%).

\begin{tabular}{|c|c|c|c|c|c|c|c|c|c|c|}
\hline$\widehat{L}\left(\mathscr{A}_{\mathcal{H}^{*}}\left(\mathcal{D}_{n}\right)\right)$ & \begin{tabular}{|c|} 
Loss \\
Function
\end{tabular} & ORF & $\begin{array}{l}\text { S1 } \\
\text { RFR }\end{array}$ & PRF & ORF & $\begin{array}{l}\text { S2 } \\
\text { RFR }\end{array}$ & PRF & ORF & $\begin{array}{l}\text { S3 } \\
\text { RFR }\end{array}$ & RF \\
\hline TR & & $3.9+0.4$ & $3.1+0.3$ & $2.7+0.3$ & $10.2+1.0$ & $\overline{4.0+0.4}$ & $3.1 \pm 0.4$ & $12.9+1.4$ & $3.9+0.4$ & $3.7 \pm 0.4$ \\
\hline $\mathrm{H}$ & $\ell$ TRAE & 3. & $3.0 \pm 0.3$ & 2.7 & & & & & & \\
\hline & $\ell_{T R}^{T R}$ & 0.8 & 12.2 & 2.9 & 37. & & 5 & $=3.7$ & & $.4 \pm 0.5$ \\
\hline & $\ell_{\mathrm{TR}}^{\mathrm{TR}}$ & & & & 12. & & & 1.2 & & \\
\hline & $\ell_{\mathrm{H}}$ & & & & & & & & 0.7 & \\
\hline HDD & $\ell_{\mathrm{H}}$ & $8.2 \pm 0.9$ & 6 & & & & & & 0.9 & .9 \\
\hline
\end{tabular}

\begin{tabular}{|c|c|c|c|c|c|c|c|c|c|c|}
\hline$L\left(\mathscr{A}_{\mathcal{H}^{*}}\left(\mathcal{D}_{n}\right)\right)$ & \begin{tabular}{||c|} 
Loss \\
Function
\end{tabular} & ORF & $\begin{array}{l}\text { S1 } \\
\text { RFR }\end{array}$ & PRF & ORF & $\begin{array}{l}\text { S2 } \\
\text { RFR }\end{array}$ & PRF & $\sigma_{K F}$ & $\begin{array}{l}\text { S3 } \\
\text { RFR }\end{array}$ & $2 \mathrm{~F}$ \\
\hline TI & $\begin{array}{l}t \\
\end{array}$ & 6 & $5.8 \pm 0.5$ & $5.2 \pm$ & $2 \pm 1.3$ & $.1 \pm 0.7$ & & & 7. & \\
\hline & & & 5. & & & & & & & \\
\hline TFM & & $\begin{array}{l}36.8 \pm 3.7 \\
7.1+0.6\end{array}$ & $\begin{array}{r}16.5 \pm 1.7 \\
5.8+0.6\end{array}$ & 5.8 & $\begin{array}{l}43.0 \pm 3.7 \\
16.5+16\end{array}$ & $19.8 \pm 2.0$ & & $\begin{array}{l}42.0 \pm 4.6 \\
17.0 \pm 1.8\end{array}$ & $\begin{array}{r}19.2 \pm 1.9 \\
8.3+0.9\end{array}$ & 9 \\
\hline 1 & ${ }^{{ }^{t} \mathrm{~T}} \mathrm{~T}_{\ell}$ & $10.7=$ & $\begin{array}{l}8.0 \\
8.9\end{array}$ & 7.2. & $\begin{array}{l}10.0 \\
14.8\end{array}$ & $9.5 \pm 0.9$ & & $\begin{array}{l}17.0 \\
32.1\end{array}$ & $\begin{array}{l}0.0 \pm 0.9 \\
11.9 \pm 1.3\end{array}$ & $\begin{array}{l}.3 \pm 0.8 \\
.1 \pm 0.8\end{array}$ \\
\hline $\mathrm{HD}$ & & & 0.9 & & $9 I 1$ & & & 3. & & $.1 \pm 0.8$ \\
\hline
\end{tabular}

\section{Conclusions}

In this paper, authors developed a series of data-driven models able to estimate the vessel safety properties during the preliminary design stages. In particular, authors have proposed a vessel crash stop maneuvering performance prediction which can accurately predict the results of this safety test. To achieve this goal, authors proposed to use a recent improvement of the RF learning algorithm and show that an accurate tuning procedure can remarkably improve their predictive power. Results on real-world data, collected and provided by the DAMEN Shipyards, demonstrate the effectiveness of the proposal which is already exploited in DAMEN for the realization of new High-Speed Craft vessels. This work is a step forward in the direction of a smart and safe ship design since it allows to better forecast the safety properties of a ship before its production. As a future work, authors plan to derive new predictive models able to both take into account the principal equation methods and the data driven ones, analogously to [6], to obtain even more accurate models able to provide more insights on the ship safety properties. 


\section{References}

1. ABS: Guide for vessel manoeuvrability (2006)

2. Anguita, D., Ghio, A., Oneto, L., Ridella, S.: In-sample and out-of-sample model selection and error estimation for support vector machines. IEEE TNNLS 23(9), 1390-1406 (2012)

3. Blaser, R., Fryzlewicz, P.: Random rotation ensembles. JMLR 2, 1-15 (2015)

4. Breiman, L.: Random forests. Machine learning 45(1), 5-32 (2001)

5. Catoni, O.: Pac-Bayesian Supervised Classification. Institute of Mathematical Statistics (2007)

6. Coraddu, A., Oneto, L., Baldi, F., Anguita, A.: Ship efficiency forecast based on sensors data collection: Improving numerical models through data analytics. In: OCEANS 2015-Genova (2015)

7. Coraddu, A., Oneto, L., Baldi, F., Anguita, D.: Vessels fuel consumption forecast and trim optimisation: A data analytics perspective. Ocean Engineering 130, 351$370(2017)$

8. Damen: http://products.damen.com/en/clusters/crew-supply-vessel

9. Donders, A.R.T., van der Heijden, G.J.M.G., Stijnen, T., Moons, K.G.: Review: a gentle introduction to imputation of missing values. Journal of clinical epidemiology 59(10), 1087-1091 (2006)

10. Fernández-Delgado, M., Cernadas, E., Barro, S., Amorim, D.: Do we need hundreds of classifiers to solve real world classification problems? JMLR 15(1), 3133-3181 (2014)

11. Germain, P., Lacasse, A., Laviolette, A. ahd Marchand, M., F., R.J.: Risk bounds for the majority vote: From a pac-bayesian analysis to a learning algorithm. JMLR 16(4), 787-860 (2015)

12. Harvald, S.A.: Factors affecting the stopping ability of vessels. Journal of International Shipbuilding Progress 23(260), 106-121 (1976)

13. Hoeffding, W.: Probability inequalities for sums of bounded random variables. Journal of the American statistical association 58(301), 13-30 (1963)

14. Hoppe, H.: International regulations for high-speed craft an overview. In: Int. Conf. on Fast Sea Transportation (2005)

15. IMO: Maritime safety, http://www.imo.org/

16. IMO: Resolution MSC.36(63), Int. Code of Safety for High-Speed Craft (1994)

17. IMO: Resolution MSC.97(73), Int. Code of Safety for High-Speed Craft (2000)

18. Langxiong, G., Liangming, L., Yuangzhou, Z., Baogang, Z.: A new method for accurate prediction of ship's inertial stopping distance. Research Journal of Applied Sciences, Engineering and Technology 18(6), 3437-3440 (2013)

19. Lever, G., Laviolette, F., Shawe-Taylor, F.: Tighter pac-bayes bounds through distribution-dependent priors. Theoretical Computer Science 473, 4-28 (2013)

20. Ming, L., Liu, J.X., Yang, S.: A new method on calculation of vessels stopping distance and crash stopping distance. In: Advanced Materials Research. vol. 779, pp. 800-804 (2013)

21. Okamoto, H., Tanaka, A., Nozawa, K., Saito, Y.: Stopping abilities of vessels equipped with controllable pitch propeller. Journal of International Shipbuilding Progress 21(235), 53-69 (1974)

22. Orlandi, I., Oneto, L., Anguita, D.: Random forests model selection. In: ESANN (2016)

23. Vapnik, V.N.: Statistical learning theory. Wiley (1998)

24. Wainberg, M., Alipanahi, B., Frey, B.J.: Are random forests truly the best classifiers? JMLR 17(110), 1-5 (2016)

25. Wirz, D.I..F.: Optimisation of the crash-stop manoeuvre of vessels employing slowspeed two-stroke engines and fixed pitch propellers. Journal of Marine Engineering \& Technology 11(1), 35-43 (2012) 